\title{
Evaluación de actividades académicas, investigativas y de proyección social desarrolladas por el programa de ingeniería biotecnológica como aporte a su proceso de autoevaluación con fines de acreditación
}

Ana María Arismendy Pabón ${ }^{1}$, Ingrid Liliana González Jáuregui ${ }^{2}$, Nohora Milena López Rico ${ }^{3}$, Yaneth A. Muñoz Peñaloza ${ }^{4}$

\section{Resumen}

El estudio se enfocó en la evaluación de las actividades académicas, investigativas y de proyección social desarrolladas en el programa de Ingeniería Biotecnológica desde los requerimientos del Consejo Nacional de Acreditación (CNA), y los aspectos evaluados fueron: convenios, trabajos de grado, prácticas profesionales, grupos y semilleros de investigación y egresados, en el periodo 2005-2010. Se recolectó información de estos aspectos, se generaron documentos a partir de la información solicitada por el CNA, se realizaron los análisis de los indicadores seleccionados y se determinó su grado de cumplimiento. Para los convenios, trabajos de grado y la práctica profesional el cumplimiento fue satisfactorio. El programa cuenta con el apoyo de 2 grupos de investigación y 8 semilleros, siendo suficiente en el cumplimiento del indicador. Los indicadores de egresados no tuvieron cumplimiento, debido a que se requería la totalidad de la información, se obtuvo un 40,7\% de los registros. Se diseñó un plan estratégico para la actualización y disponibilidad de la información del Programa, generar un proceso de cambio con énfasis en el mejoramiento continuo y el logro de la acreditación de la Carrera.

Palabras claves: Indicadores, Consejo Nacional de Acreditación, Acreditación, Plan estratégico.

\section{Abstract}

The study focused on the evaluation of academic activities, research and social outreach program developed in Biotechnological Engineering from the requirements of the National Council of Accreditation (CNA), and the aspects evaluated were: agreements, grade work, internships groups and seed research and graduates, in the period 2005-2010. Information was collected from these aspects, documents generated from the information requested by the CNA; it made ??the analysis of selected indicators and determined the degree of compliance. For conventions, degree and work practice compliance was satisfactory. The program is supported by two research groups and 8 seed, is sufficient in meeting the indicator. The graduation rates did not comply, because

\footnotetext{
${ }^{1}$ Estudiante de Ingeniería Biotecnológica, Universidad Francisco de Paula Santander. Código 1610319, mary_aris_20@hotmail.com

2 Estudiante de Ingeniería Biotecnológica, Universidad Francisco de Paula Santander. Código 1610235, lilianagonzalezja@hotmail.com

${ }^{3}$ Estudiante de Ingeniería Biotecnológica, Universidad Francisco de Paula Santander. Código 1610068, nohoralor@hotmail.com

${ }^{4}$ Directora Ingeniería Biotecnológica, Directora del proyecto, Universidad Francisco de Paula Santander. yamp92@hotmail.com
} 
all required information was obtained $40.7 \%$ of the records. We designed a strategic plan for updating and availability of program information; generate a process of change with an emphasis on continuous improvement and achieving accreditation from the Carrera.

Keywords: Indicators, National Accreditation Council, Accreditation, Strategic Plan.

\section{Introducción}

$\mathrm{L}$ os programas académicos e las instituciones de educación superior pueden aspirar a la acreditación a través de un mejoramiento continuo, reconocimiento otorgado por el Ministerio de Educación Nacional (MEN), sobre la calidad de un programa o de una institución basándose en un proceso previo de autoevaluación. La universidad Francisco de Paula Santander (UFPS) reconoce que la acreditación contribuye al mejoramiento de los Programas Académicos, desarrollando autoevaluación permanente; ejecutando planes de mejoramiento, siendo un punto de partida para afianzar la autonomía universitaria.

El plan de estudios de Ingeniería Biotecnológica de la UFPS en el año 2005 recibió por parte del MEN el registro calificado al cumplir con los estándares mínimos de calidad requeridos y por un período de siete años.

El presente estudio comprendió la recopilación y organización de la información de las actividades académicas, investigativas y de proyección social desarrolladas por el programa en el periodo 20052010, también el cumplimiento de la calidad académica desde los indicadores del CNA y el diseño de un Plan Estratégico para mejorar el manejo de la información, su organización, actualización y disponibilidad a la comunidad en general

\section{Materiales y métodos}

El estudio se realizó en la Universidad Francisco de Paula Santander en el Programa académico Ingeniería Biotecnológica en cuatro etapas. Se inició con la recolección de la información sobre convenios, trabajos de grado, la Práctica profesional, las actividades investigativas y los egresados; examinando el archivo del programa y consultando dependencias de la Universidad como la Facultad, la Secretaría General y la Biblioteca. También se inspeccionó la pág. web de la universidad y la Facultad. Seguido según los aspectos de estudio, se seleccionaron 18 indicadores del CNA que su evaluación. Se continuó con la organización y procesamiento de la información, diseñándose formatos según los indicadores del CNA. También se elaboraron encuestas para egresados y docentes y se diseñó el directorio de egresados. Se continuó con el análisis y evaluación de la información para determinar el cumplimiento de los indicadores teniendo en cuenta la escala: total, satisfactorio, suficiente, escaso y no cumple. Finalmente se diseñó un plan estratégico con el fin de mantener actualizada y disponible la información de la Carrera para la comunidad en general.

\section{Resultados y discusiones}

La información sobre Convenios, Trabajos de Grado, Práctica profesional, Grupos y Semilleros de Investigación y los egresados en los últimos seis años se encontraba dispersa en diferentes dependencias de la Universidad, pero la mayor parte en el archivo de la Carrera.

La organización de la información realizada a través de los formatos diseñados según el CNA para convenios, trabajos de grado y práctica profesional, se constituyen documentos soportes del estudio. La recopilación de la información sobre investigación comprende unos documentos soporte. Con respecto a la información de los egresados se realizó un directorio y se diseñó una encuesta. Para la organización en general de la información del Programa (Archivo), se realizó según la tabla de retención Documental de la UFPS. Con la información recolectada y organizada de la Carrera, se procedió a su evaluación y emisión de juicios.

Los resultados de la evaluación de los indicadores del CNA, según escala determinada, se presentan a continuación. 
Convenios. Se evaluaron los convenios establecidos por el Programa en el periodo 2005-2010, según el Factor, la Característica y el indicador señalados por el CNA.

Factor 3- Profesores, Característica 14- Interacción con las comunidades académicas, Indicador a) Número de convenios activos de nivel nacional e internacional que han propiciado la efectiva interacción académica de los profesores del programa.

Las actividades desarrolladas por los docentes del programa han sido producto de la gestión institucional y personal. Los convenios marco con la Universidad de los Andes y la Universidad Experimental del Táchira UNET (San Cristóbal- Venezuela) pudieron contribuir con el desarrollo de las maestrías. Los docentes han dirigido trabajos de grado con la firma de convenios, pero su registro solo se realizó a partir del año 2008. La encuesta aplicada contribuyó con la información del indicador. Cumplimiento del indicador: suficiente.

Factor 4 - Procesos Académicos, Característica 19. Integridad del Currículo, Indicador a: número de convenios establecidos por la institución que garanticen la movilidad estudiantil con otras instituciones nacionales e internacionales.

El cumplimiento del indicador fue satisfactorio debido a que en los últimos cinco años se han generado convenios con nuevas instituciones y se han renovado los existentes, permitiendo una constante movilidad estudiantil, ver Gráfica No 1.

Gráfica 1. Comportamiento del desarrollo de convenios IB, periodo 2005-2010.

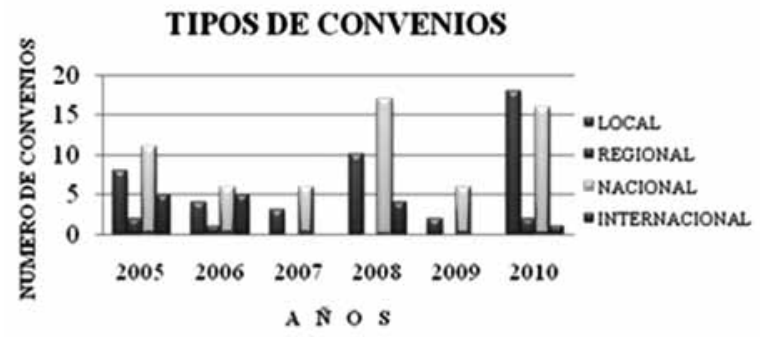

Factor 4 - Procesos Académicos, Característica 21- Relaciones nacionales e internacionales del programa, Indicador $b$. Número de convenios suscritos y actividades de cooperación académica desarrolladas por el programa con instituciones y programas de alta calidad, acreditados por entida- des de reconocida legitimidad nacional e internacional.

Se obtuvo el número total de convenios realizados por el programa con instituciones y programas de alta calidad, teniendo en cuenta los Trabajos de Grado y las Prácticas profesionales, fueron 29 convenios para Prácticas valor mayor al total de los convenios para Trabajo de Grado de 25 convenios. Cumplimiento del indicador: satisfactorio.

Factor 4 - Procesos Académicos, Característica 21- Relaciones nacionales e internacionales del programa, Indicador c. Proyectos desarrollados en la institución como producto de la gestión realizada por directivos, profesores y estudiantes del programa, a través de su participación en actividades de cooperación académica con miembros de comunidades nacionales e internacionales de reconocido liderazgo en el área del programa.

Gráfica 2. Proyectos de cooperación académica con entidades de alta calidad.

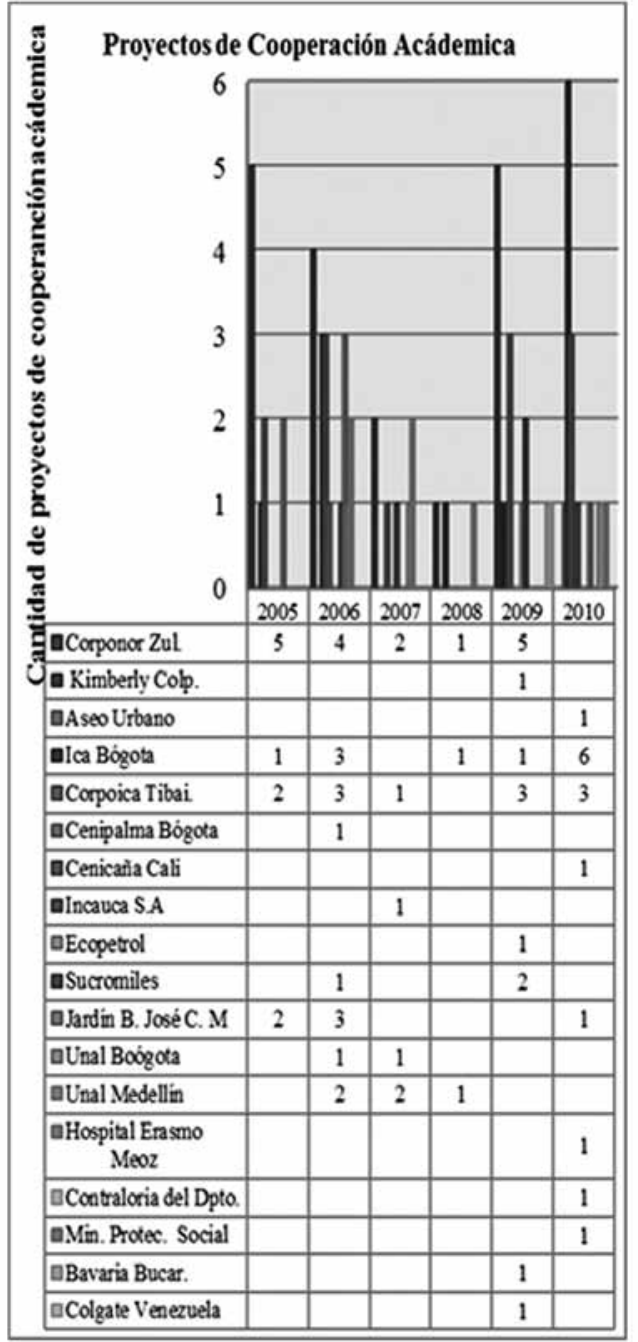


En el cumplimiento de este indicador se mostró el número de trabajos de grado realizados en las diferentes entidades de reconocido liderazgo o de alta calidad, véase Gráfica 2.

Según los resultados obtenidos en la gráfica anterior se puede señalar que las empresas en las que más se han realizados trabajos de cooperación académica son CORPONOR Zulia, ICA y CORPOICA TIBAITATÁ- Bogotá, con las otras entidades se han realizado trabajos en menor proporción. Cumplimiento del indicador: satisfactorio. Para el mejoramiento de este indicador se debe reforzar la relación entidad - estudiante a nivel local, nacional e internacional con empresas de alta calidad en las cuales se haya realizado pocos trabajos de grado, con el fin de aumentar el número de proyectos y crear convenios marco que permitan que los estudiantes realicen sus trabajos de grado, prácticas profesionales o desarrollar capacitaciones, para contribuir a la acreditación del programa. Cumplimiento del indicador: satisfactorio.

Factor 4 - Procesos Académicos, Característica 21- Relaciones nacionales e internacionales del programa, Indicador e. Porcentaje de directivos, profesores y estudiantes del programa que en los últimos cinco años, han participado en actividades de cooperación académica con miembros de comunidades nacionales e internacionales de reconocido liderazgo en el área del programa. Resultados efectivos de dicha participación para el programa.

Para el cumplimiento de este indicador se establecieron dos grupos participativos en actividades de cooperación académica que son los profesores, quienes son los encargados de ejercer como jurados calificadores, generalmente son tres y han participado durante un largo tiempo en el programa de Ingeniería Biotecnológica, y finalmente los estudiantes que son los encargados de realizar sus trabajos de grado. En este indicador no se aplicó el término directivos debido a que los trabajos de grado fueron dirigidos por miembros directos de las mismas entidades, y por lo tanto no se generaron directivos por parte de la Universidad Francisco de Paula Santander.
Gráfica 3. Proyectos de Ingeniería Biotecnológica con carácter social.

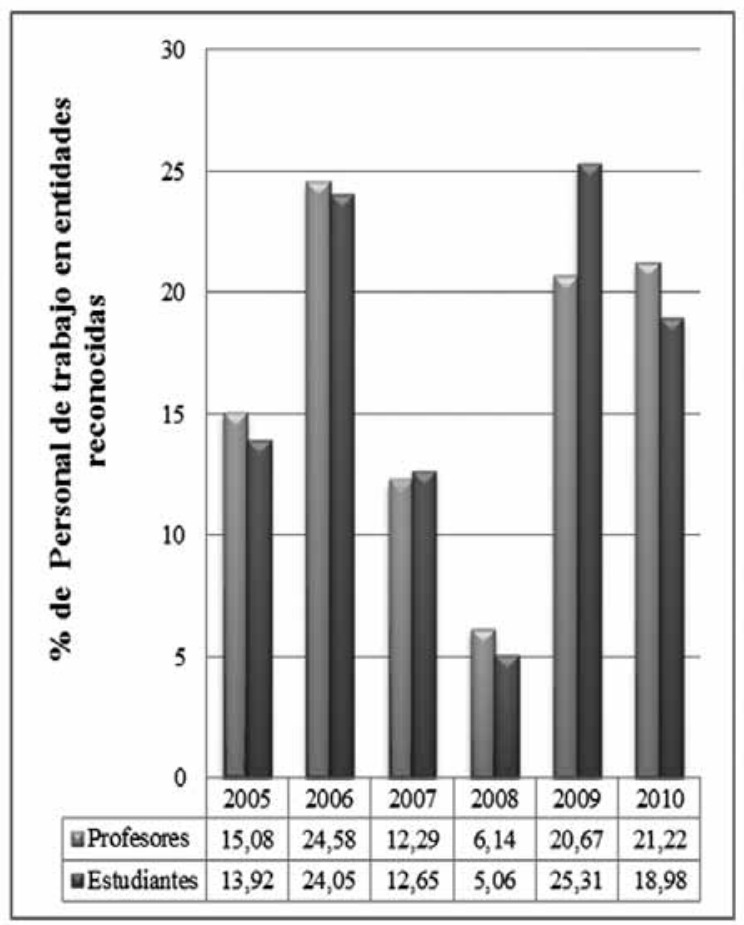

En el análisis del cumplimiento del indicador; se observó mediante la gráfica anterior que el porcentaje de profesores y estudiantes fue muy bajo en los años 2007 y 2008, especialmente en el año 2008, esto indica que en este año se generaron pocos trabajos de grado con dichas entidades, probablemente porque los estudiantes se ubicaron en entidades locales y nacionales que brindaron nuevas oportunidades pero no necesariamente son reconocidas. En el año 2006 el porcentaje de los participantes estuvo casi en los 2 casos alrededor del $25 \%$, lo que indica que la cantidad de participantes estuvo aproximadamente constante; en el año 2005 la cantidad de profesores estuvo casi por el $15 \%$ y los estudiantes cerca de un $14 \%$; debido a que existen trabajos de grado a los cuales se les otorga en la mayoría de casos 3 profesores que sirven como jurado calificador; en el año 2009 se generó un gran aumento de estudiantes debido a que existen trabajos de grado en los cuales laboran máximo 3 estudiantes. En el año 2010 se observa un aumento un poco significativo de profesores se generaron más trabajos con 3 jurados que con dos jurados, por esta razón se observa el crecimiento de la columna profesores. Cumplimiento del indicador: satisfactorio.

Trabajo de Grado y Práctica Profesional. Se de- 
terminó la información y se realizó el análisis de cumplimiento según los indicadores.

Factor 1 - Misión Y Proyecto Institucional, Características 4-Relevancia Académica y Pertinencia Social del Programa, Indicador d.

Número y tipo de proyectos de carácter social que adelanta el programa mediante sus funciones de docencia, extensión e investigación.

Para llevar a cabo la evaluación de este indicador se utilizó como base 216 trabajos de grado pertenecientes al programa de Ingeniería Biotecnológica, que comprenden la modalidad extensión y la modalidad investigación periodo 2005-2010. Se incluyeron pasantías, trabajos dirigidos y tesis investigativas. El primer grupo corresponde a pasantías y trabajos dirigidos (T.D) que son modalidad extensión y segunda por investigación que incluye las tesis desarrolladas por los estudiantes del programa durante los últimos 6 años, como requisito para obtener el título de ingeniero biotecnológico, ver Gráfica 4.

Gráfica 4. Proyectos de Ingeniería Biotecnológica con carácter social

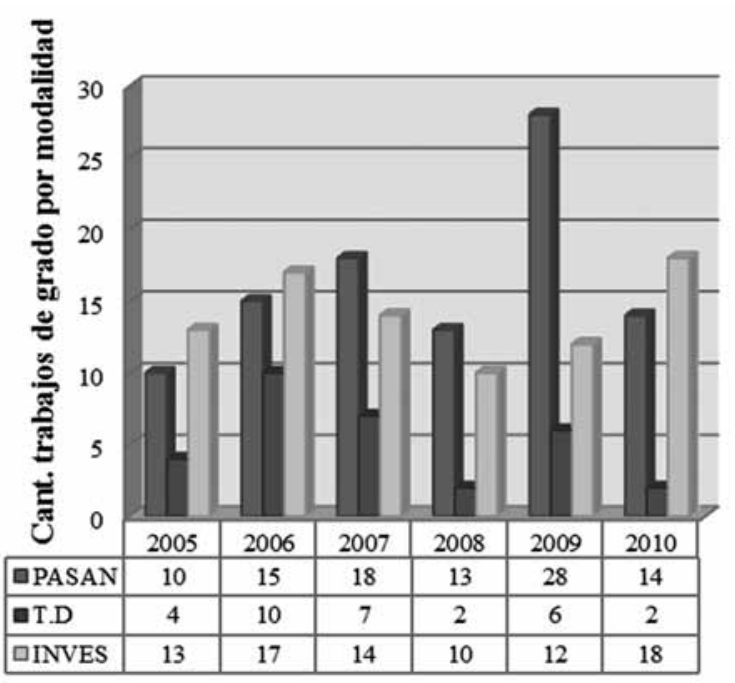

Los proyectos de grado en la modalidad extensión comprendida por las pasantías con un (46\%) y trabajos dirigidos (14.55\%), suman un $60.55 \%$, y la investigación comprendida por las tesis un $39.43 \%$, lo cual permite determinar que el programa ha trabajado ampliamente en la extensión o trabajo social, que está estrechamente relacionado con las empresas principalmente en el caso de las pasantías y los trabajos dirigidos, ver Gráfica 5. Cumplimiento del indicador: satisfactorio.
Gráfica 5. Porcentaje de trabajo de grado modalidad extensión e investigación.

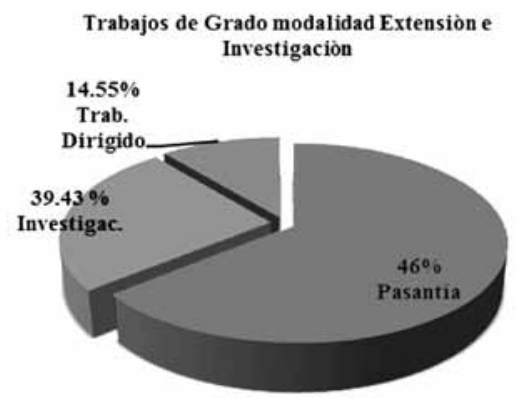

Factor 4 - Procesos Académicos, Característica 24-Relaciones nacionales e internacionales del programa, Indicador c. Número y título de trabajos realizados por estudiantes del programa en los últimos cinco años que han merecido premios o reconocimientos significativos de parte de la comunidad académica nacional o internacional.

La Universidad Francisco de Paula Santander, reconoce los trabajos de grado de forma significativa de la siguiente manera, con calificación de 4.5 hasta 4.9 meritorio y si la calificación es de 5.0 es un proyecto de grado laureado.

Según los resultados mostrados en la Gráfica 6 y la Tabla 1 , se puede afirmar que el reconocimiento laureado le fue otorgado a 1 proyecto de grado en el año 2005 es decir calificación de 5.0, para el reconocimiento de trabajo de grado meritorio obtuvo un índice muy bajo en el año 2006 de 4.7\% , y el resto de los años se mantuvo en un rango del $18.5 \%$ al $40.7 \%$,lo que indica que aunque este incentivo por parte de la Universidad es exigente, se cuenta con un buen número (53) de proyectos de grado meritorios. Cumplimiento del indicador: satisfactorio.

Gráfica 6. Reconocimientos en trabajos de grado.

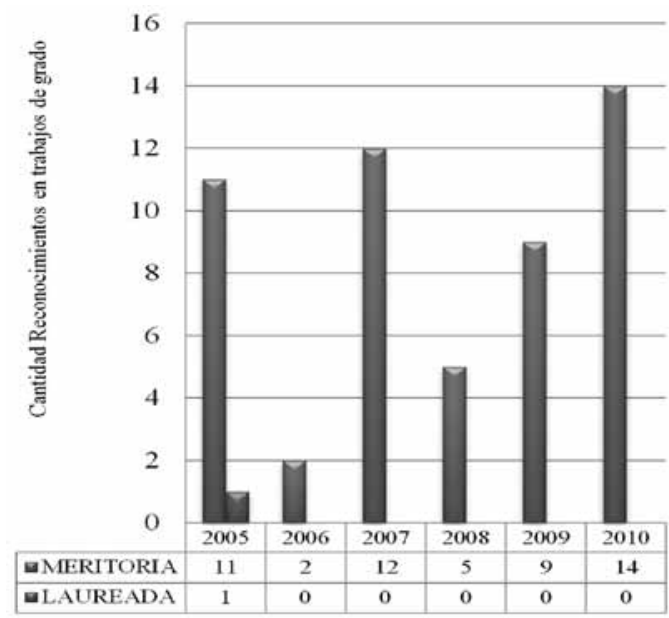


Tabla 1. Porcentaje de reconocimientos de trabajos de grado $2005-2010$

\begin{tabular}{|c|c|c|c|c|c|c|c|c|c|c|c|c|}
\hline \multirow{2}{*}{$\begin{array}{c}\text { ANo } \\
\text { Reconocimiento }\end{array}$} & \multicolumn{2}{|c|}{2005} & \multicolumn{2}{|c|}{2006} & \multicolumn{2}{|c|}{2007} & \multicolumn{2}{|c|}{2008} & \multicolumn{2}{|c|}{2009} & \multicolumn{2}{|c|}{2010} \\
\cline { 2 - 14 } & $\mathrm{N}^{0}$ & $\%$ & $\mathrm{~N}^{0}$ & $\%$ & $\mathrm{~N}^{0}$ & $\%$ & $\mathrm{~N}^{\circ}$ & $\%$ & $\mathrm{~N}^{0}$ & $\%$ & $\mathrm{~N}^{\circ}$ & $\%$ \\
\hline Laureado & 1 & 3.7 & 0 & 0 & 0 & 0 & 0 & 0 & 0 & 0 & 0 & 0 \\
\hline Meritorio & 11 & 40.7 & 2 & 4.7 & 12 & 25.5 & 5 & 18.5 & 9 & 20 & 14 & 37.8 \\
\hline
\end{tabular}

Factor 4 - Procesos Académicos Característica 28-Extensión o proyección social, Indicador e. Número y tipo de proyectos y actividades de extensión o proyección a la comunidad que ha desarrollado el programa en los últimos cinco años.

Según los datos obtenidos, la gráfica 7 muestra que el trabajo de proyección a la comunidad en el cual se incluyen los trabajos de grado de modalidad pasantía y trabajo dirigido, y la materia de práctica profesional; en el periodo 2005-2010, muestra un buen número de pasantías y prácticas profesionales en comparación a proyectos dirigidos.

Gráfica 7. Comportamiento de la extensión en IB, periodo 2005-2010.

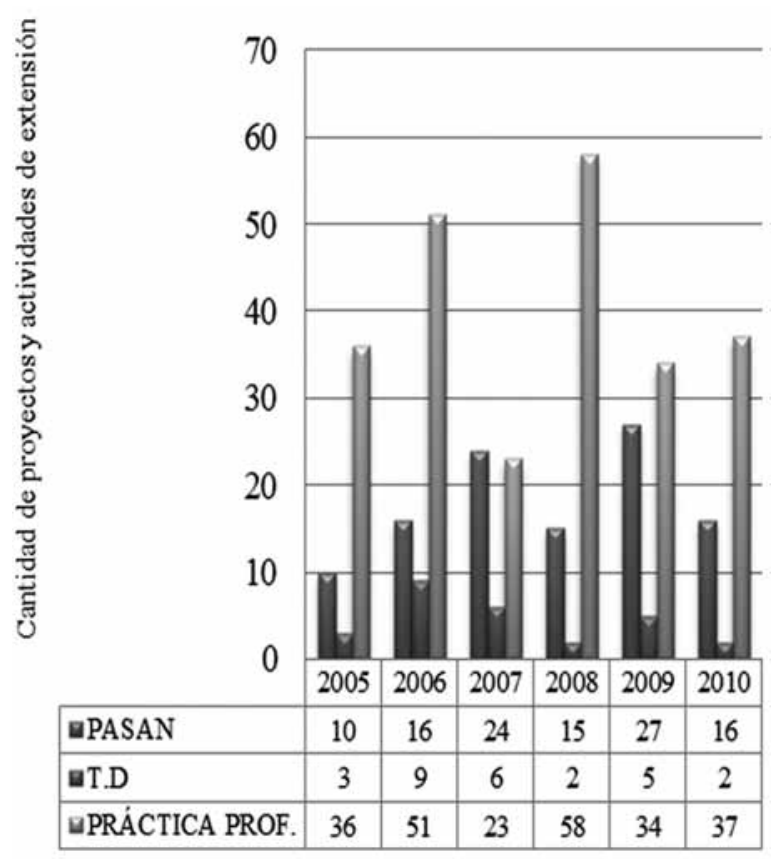

Se puede apreciar que se ha generado un gran número de trabajos de proyección social, según las cifras de la gráfica 7, especialmente con la práctica profesional (asignatura que se lleva a cabo con las Empresas), este trabajo ha permitido que los estudiantes muestren sus capacidades y conocimientos a las diferentes entidades o empresas, en lo con- cerniente a los campos de acción del programa de Ingeniería Biotecnológica, dándolo a conocer. Los resultados muestran un fortalecimiento de la proyección social de la Carrera. Cumplimiento del indicador: satisfactorio.

Factor 4 - Procesos Académicos, Característica 28-Extensión o proyección social, Indicador d. Impacto que han tenido en el entorno los resultados de los proyectos de extensión o proyección social desarrollados por el programa.

Los proyectos se han realizado según las líneas de investigación que son: línea 1 desarrollo y manejo de procesos y cadenas productivas agropecuarias, línea 2 productos de interés comercial, línea 3 bioprospección y línea 4 biotecnología ambiental; por lo que se puede afirmar que se han desarrollado todas las líneas que incluyen las diferentes especialidades del programa de Ingeniería Biotecnológica. El impacto de cada trabajo de grado que corresponde a los aportes relevantes (protocolos, procedimientos y otros) y los resultados significativos, información importante para las empresas. Igualmente la práctica profesional, ha aportado en el impacto del programa con su entorno en la medida que ha sido el primer contacto de los estudiantes con el sector externo y que debido a su buen desempeño se ha podido continuar o con el desarrollo de los trabajos de grado en especial las pasantías. Cumplimiento del indicador: satisfactorio.

Investigación del Programa. Se analizó la investigación del Programa en el periodo 2005-2010, según el Factor, la Característica y el indicador señalador por el CNA, de la siguiente manera.

Factor 4 - Procesos Académicos, Característica 27. Compromiso con la investigación, Indicador d. Número de proyectos que evidencien la articulación de la actividad investigativa de los profesores del programa con sus actividades de docencia y de extensión o proyección social.

La información necesaria para el indicador requiere de los formatos trabajos de grado, el cuadro de los proyectos financiados por el FINU u otras dependencias de la universidad que apoyan la investigación, resultados de una encuesta corta que se aplicó a los profesores para completar la información, el documento soporte cual presenta toda la información sobre investigación de la Carrera 
incluyendo las jornadas de acreditación 2010, en el cual se evaluaron los indicadores asociados a la investigación incluyéndose el indicador d del Factor 4, característica 27 contemplado de manera específica en el presente estudio.

Mediante la información de los semilleros y grupos de investigación que apoyan el Programa Ingeniería Biotecnológica, se determinó que los semilleros SIBBA y SINBI poseen el mayor número de proyectos terminados, el semillero SEIBA es el único que posee proyectos formulados, los otros semilleros poseen muy pocos proyectos terminados y del semillero SICTA no se posee información sobre los proyectos que ha realizado o formulado. Sobre el Grupo Ambiente y Vida se tiene 18 proyectos periodo 2005-2010. El grupo GIBA posee 6 proyectos terminados y se ha integrado al grupo AMBIENTE Y VIDA, del grupo MAJUMBA no se tiene información sobre los proyectos. También se determinó que los docentes han contribuido en gran parte a motivar a los estudiantes a participar en actividades investigativas como son los semilleros; por lo cual se ha mostrado que la carrera la apoyan 8 semilleros de investigación. Cumplimiento del indicador: suficiente.

Egresados del programa. Se presenta el análisis de los egresados del Programa Ingeniería de Producción Biotecnológica e Ingeniería Biotecnológica en el periodo 2001-2010, según el Factor, la Característica y el indicador señalador por el CNA, de la siguiente manera.

Factor 7 - Egresados y Articulación con el medio, Característica 37-Influencia del programa en el medio, Indicador d. Información estadística sobre el impacto social de los proyectos que el programa desarrollo o contribuyó a desarrollar en los últimos cinco años.

El impacto generado por los trabajos de grado se analizó a partir de las líneas-áreas de investigación y las necesidades de los sectores productivos. El programa en los últimos cinco años ha impactado en las actividades ambientales comprometidas con la protección del recurso hídrico y el manejo de residuos sólidos, seguido del aporte al control de calidad microbiológica de procesos y el desarrollo de los bioprocesos con un 20\%. El trabajo conjunto entre áreas como la biotecnología agrícola, ambiental y la molecular han ampliado más el campo de acción de la Ingeniería biotecnológica, ver Gráfica 8. Cumplimiento del indicador: satisfactorio.

Gráfica 8. Impacto social de los proyectos realizados en el programa.

Áreas de investigación

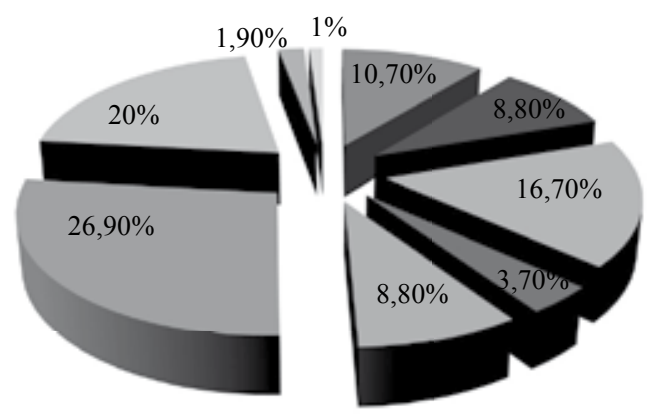

$\begin{array}{ll}\square \text { B. Agrícola-B. Ambiental } ₫ \text { B. Agrícola-B.Molecular } \\ \square \text { B. Vegetal } & \square \text { Agrícola-Bioprospección } \\ \square \text { B. Agrícola } & \square \text { B. Ambiental } \\ \square \text { B. Industrial } & \square \text { B. Animal } \\ \square \text { Otros } & \end{array}$

Factor 7 - Egresados y Articulación con el medio, Característica 38-Impacto de los egresados en el medio social y académicos, Indicador a. Existencia de registros completos y actualizados sobre ocupación y ubicación profesional de los egresados del programa.

La recolección de la información tuvo como resultado la obtención del 41,42\% de los registros actualizados de los egresados del programa. No se cumple por falta de información.

Factor 7 - Egresados y Articulación con el medio, Característica 38-Impacto de los egresados en el medio social y académicos Indicador $b$. Grado de correlación existente entre la ocupación y perfil profesional de los egresados y el perfil de formación del programa.

De acuerdo a la muestra obtenida se pudo establecer que el $61,7 \%$ de los egresados ocupan un cargo de acuerdo al perfil del programa, el 15,6\% laboran como independientes o fuera del perfil de formación, el $10.15 \%$ de los egresados están realizando estudios de postgrado, el 5,46\% están realizando estudios de postgrado y además se encuentran laborando de acuerdo al perfil, el 0,78\% 
están realizando estudios de postgrado y trabajan como independiente, el 0,78\% está realizando estudios de pregrado y el $5,46 \%$ no se encuentra desarrollando alguna actividad. Véase Gráfica 9. Cumplimiento del indicador: escaso.

Gráfica 9. Correlación entre la Ocupación y perfil profesional de egresados.

\section{Relación egresado-ocupación}

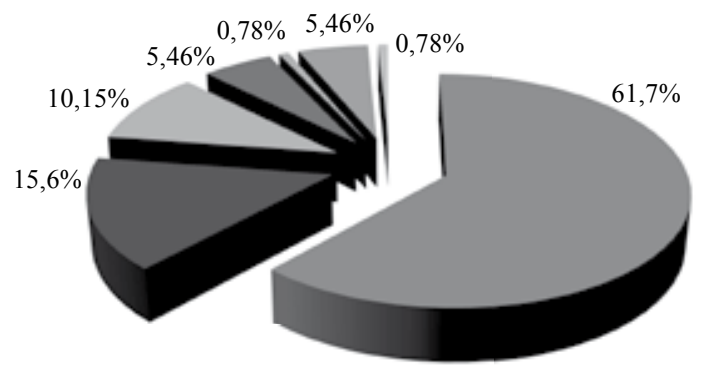

$\square \mathrm{TP} \square \mathrm{TI} \backsim \mathrm{EP} \backsim \mathrm{EP}-\mathrm{TP} \square \mathrm{EP}-\mathrm{TI} \square \mathrm{N} \backsim \mathrm{PRE}$

Factor 7 - Egresados y Articulación con el medio, Característica 38-Impacto de los egresados en el medio social y académicos, Indicador c. Apreciación de los egresados, empleadores y usuarios externos sobre la calidad de la formación recibida en el programa.

De la encuesta y el formato aplicado a los egresados, se obtuvo respuesta del $26,2 \%$ de la población teniéndose como muestra 81 encuestas diligenciadas. 54 egresados consideran que la calidad de la formación recibida en el programa es buena $(66,66 \%)$, seguido de 16 egresados que consideran una calidad media $(19,75 \%), 8$ responden que es excelente $(9,87 \%)$ y 3 consideran que la calidad de la formación es baja (3.7\%). Cumplimiento del indicador: escaso.

Factor 7 - Egresados y Articulación con el medio, Característica 39- Impacto de los egresados en el medio social y académico, Indicador a. Índice de empleo entre los egresados del programa.

Se obtuvo respuesta de 128 egresados del programa. Con esta información se obtuvo el índice de empleo dentro de los egresados (83,59\%), obteniendo que 107 se encuentran laborando, 13 están realizando estudios de postgrado, uno está estudiando Pregrado y 7 no están desarrollando ninguna actividad. Cumplimiento del indicador: escaso.

Factor 7 - Egresados y Articulación con el medio, Característica 39. Impacto de los egresados en el medio social y académico, Indicador b. Porcentaje de los egresados del programa que forma parte de comunidades académicas reconocidas, de asociaciones científicas, profesionales, tecnológicas, técnicas o artísticas, y del sector productivo y financiero, en el ámbito nacional o internacional.

A partir de los resultados obtenidos de la encuesta y el formato corto, un 39,66\% de los egresados hacen parte de comunidades académicas como docentes, asesores y asistentes de investigación de las mismas, además se incluye en ellos a los estudiantes de postgrado, seguido de un 33,05\% de los egresados que hacen parte de Asociaciones científicas o tecnológicas. En cuanto al sector productivo un $26,44 \%$ de los egresados pertenece a estos y un $0,82 \%$ al sector financiero, ver Grafica 10 . Cumplimiento del indicador: escaso.

Gráfica 10. Distribución de los egresados según ubicación laboral

\section{Ubicación egresados}

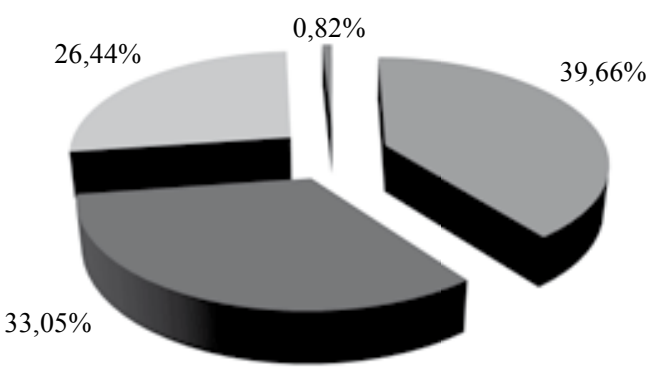

Comunidades académicas

- Aso. Cientif, Prof, Tecn o Artis.

Sector Productivo

Sector Financiero

Factor 7 - Egresados y Articulación con el medio, Característica 39. Impacto de los egresados en el medio social y académico, Indicador c. Porcentaje de egresados del programa que ha recibido distinciones y reconocimientos significativos por su desempeño en la disciplina, profesión, ocupación u oficio correspondiente. 
El 29,62\% de los egresados han recibido reconocimientos por el desempeño en sus ocupaciones, mientras que el 70,37 \% no los han recibido. Estos reconocimientos se han reflejado en premios por investigaciones y desempeño a nivel interno de las entidades donde laboran, liderando investigaciones, además de asensos y remuneraciones económicas. Cumplimiento del indicador: escaso.

Factor 7 - Egresados y Articulación con el medio, Característica 39. Impacto de los egresados en el medio social y académico, Indicador d. Apreciación de empleadores sobre la calidad de la formación y el desempeño de los egresados del Programa.

La apreciación no se pudo determinar porque las respuestas fueron escasas.

Al realizar un análisis de los resultados del cumplimiento de los indicadores evaluados en el presente estudio, de los 9 indicadores asociados a convenios, trabajos de grado y práctica profesional solo uno fue suficiente y los demás su cumplimiento fue satisfactorio. Al programa lo apoyan 2 grupos de investigación y 8 semilleros, se cumpliéndose el indicador de manera suficiente. Para los 8 indicadores de los egresados, uno fue satisfactorio, 5 presentaron nivel escaso y 2 no cumplieron, en parte debido a que no se contó con la totalidad de la información y se obtuvo un $41,42 \%$ de los registros.

De los 18 indicadores evaluados, 9 fueron calificados como satisfactorios, 2 suficientes, 5 escasos y 2 no cumplieron; por lo cual se determina que el Programa ha venido realizando una labor satisfactoria, que necesariamente debe mejorar hacia el cumplimiento de los indicadores no solo de los que fueron tenidos en cuenta en el presente estudio, sino los contemplados en la GUIA del CNA para lograr la acreditación de la Carrera.

El Plan de estratégico elaborado contempló: Introducción, Objetivos, Análisis de la situación, Diagnostico el cual fue realizado a través de una matriz DOFA, las estrategias y el plan de acción. El plan de acción presenta los aspectos: convenios, trabajo de grado, práctica profesional, semilleros y grupos de investigación, egresados, gestión administrativa y autoevaluación. Para cada aspecto se contempla: acciones, indicadores, fuentes, fecha y responsables.

\section{Conclusiones}

La información recolectada para convenios, prácticas profesionales y los trabajos de grado del archivo del Programa Académico IPB-IB, alguna publicada en la página www.facuagra-ufps.org; sirvió como base para diligenciar los formatos que cumplen con los indicadores del CNA.

El programa estableció buen número de convenios a nivel nacional e internacional en el periodo evaluado 2005-2010, lo cual benefició la movilidad estudiantil para los Trabajos de Grado y las Practicas Profesionales.

La carrera Ingeniería Biotecnológica ha desarrollado la formación investigativa; sin embargo no se cuenta con registros completos como evidencia en especial con respecto a la formación investigativa en el aula y Semilleros y Grupos de Investigación. Las tesis meritorias muestran un porcentaje alrededor del $20 \%$ en el periodo del estudio 2005 2010 y se considera relevante; este resultado debe impulsar a los estudiantes a realizar sus proyectos de grado de la mejor manera posible, ya que una calificación meritoria es considerada como un reconocimiento por parte de la universidad mediante el cumplimiento de los indicadores del CNA. El número de proyectos financiados por el FINU en el periodo 2005-2010 fue bajo. La consecución de la información de los semilleros y grupos de investigación no fue fácil y fue escaza.

Del total de egresados del programa se pudo obtener y actualizar un $41,4 \%$ de los registros de estos. La información se recolectó a partir de la encuesta diseñada y el formato corto que se implementó como alternativa para obtener información de la ocupación y ubicación de los egresados. La aplicación de la encuesta y el formato se realizó a partir de medios electrónicos, redes sociales, llamadas telefónicas y se hizo entrega personalmente en algunos casos. Aunque se utilizaron diferentes medios para contactar a los egresados las respuestas fueron escasas y se recibieron gradualmente.

De los indicadores evaluados se determina que el Programa ha venido realizando una labor satisfactoria, que debe mejorar hacia el cumplimiento de los indicadores no solo de los que fueron tenidos en cuenta en el presente estudio, sino todos 
los demás contemplados en la GUIA del CNA, para lograr la acreditación de la Carrera.

El plan estratégico diseñado, contribuye a la organización y actualización de la información del programa no solo la contenida en este proyecto sino la que se genere, para que el esfuerzo iniciado continúe como apoyo a la autoevaluación, mejoramiento continuo y acreditación de la Carrera.

\section{Bibliografía}

Informe de los Estándares de Calidad para la obtención del Registro Calificado Ingeniería Biotecnológica. Comité Curricular IPB-IB. (San José de Cúcuta, 2003).

MUÑOZ, Yaneth. La ingeniería Biotecnológica en la UFPS, programa pionero en Colombia y su impacto. Manizales: Memorias I congreso Iberoamericano de biotecnología y biodiversidad. 2010. P 55.

PROYECTO EDUCATIVO DE LA FACULTAD (PEF). Universidad Francisco de Paula SantanderConsejo de Facultad Ciencias Agrarias y del Ambiente. (San José de Cúcuta, 2008).

PROYECTO EDUCATIVO DEL PROGRAMA (PEP). Universidad Francisco de Paula Santander; Ingeniería Biotecnológica, Comité Curricular de Ingeniería Producción Biotecnológica- Ingeniería Biotecnológica. (San José de Cúcuta, 2009).

PROYECTO EDUCATIVO INSTITUCIONAL (PEI). Universidad Francisco de Paula SantanderConsejo Académico. (San José de Cúcuta, 2007).

SISTEMA NACIONAL DE ACREDITACIÓN. Lineamientos para la Acreditación de Programas. (Bogotá, D.C. Colombia Noviembre de 2006). 